\title{
Technology-Enabled Risk-Informed Maintenance Strategy to Minimize Operation and Maintenance Costs
}

Vivek Agarwal, Koushik Araseethota Manjunatha, James A Smith

August 2019

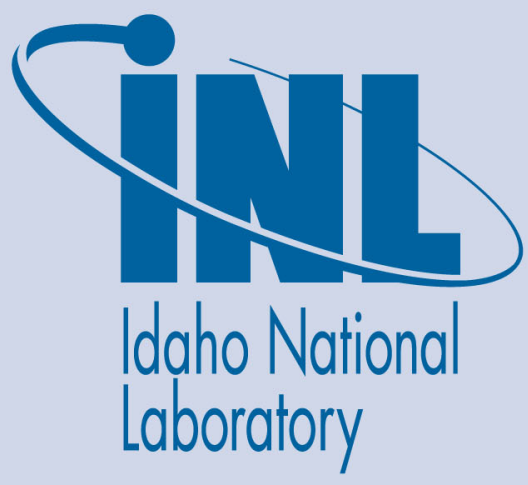

The INL is a U.S. Department of Energy National Laboratory operated by Battelle Energy Alliance 


\section{Technology-Enabled Risk-Informed Maintenance Strategy to Minimize Operation and Maintenance Costs}

Vivek Agarwal, Koushik Araseethota Manjunatha, James A Smith

August 2019

Idaho National Laboratory Idaho Falls, Idaho 83415

http://www.inl.gov

Prepared for the

U.S. Department of Energy

Under DOE Idaho Operations Office

Contract DE-AC07-05ID14517 


\title{
Technology-Enabled Risk-Informed Maintenance Strategy to Minimize Operation and Maintenance Costs
}

\author{
Vivek Agarwal, Koushik A. Manjunatha, and James A. Smith
}

The domestic fleet of nuclear power plants (NPPs) is facing a unique economic sustainability challenge in today's energy market due to high total operating costs. One of the major contributors to the total operating cost of an NPP is the operations and maintenance (O\&M) budget, which includes laborintense preventive maintenance (PM) programs. PM programs involve manually performing inspection, calibration, testing, and maintenance of plant assets at periodic frequencies, as well as time-based replacement of assets, irrespective of individual asset condition. This approach, combined with the push to achieve high capacity factors, has resulted in a labor-centric business model. It is time to transition from this labor-centric business model to a technology-centric business model that will enable an optimal maintenance strategy by eliminating unnecessary costs associated with time-based PM activities.

To enable this transition, the commercial nuclear industries are utilizing and developing reliable methodologies, based on available state of the art technologies, which facilitate assessing equipment condition and the dynamic risk of failure. The U.S. Department of Energy's Idaho National Laboratory (INL) under the Light Water Reactor Sustainability (LWRS) Program is partnering with PKMJ Technical Services and Public Service Enterprise Group (PSEG) Nuclear, LLC to develop and demonstrate a deployable risk-informed predictive maintenance strategy to eliminate unnecessary O\&M costs for an identified plant system, laying the foundation for scale-up to the entire plant. Recently developed technologies such as advanced wireless and wired sensors, data analytics, and risk assessment methodologies will support this transition. The technology-centric O\&M model will result in significant automation and lay the foundation for real-time condition assessment of plant assets, thus enabling condition-based maintenance and enhancing plant safety, reliability, and economics of operation.

The project team has identified the circulating water system (CWS) at Salem NPP as the target plant system to achieve project's goal of developing and demonstrating a deployable risk-informed predictive maintenance strategy at a commercial NPP. To support the project goal, three interrelated tasks have been identified:

1. Develop a risk-informed approach using existing plant information to optimize maintenance frequencies for the CWS.

2. Develop diagnostic and prognostic models to understand the health of the CWS and update the risk model to achieve risk-informed condition-based maintenance approach.

3. Develop and demonstrate a digital, automated platform to centralize the capabilities developed in tasks 1 and 2 in order to facilitate implementation of these technologies in industry.

\section{Approach}

Figure 1 presents a well-defined approach to achieve the primary goals of the project. The approach can be broken into three focal areas: (1) data sources and recommendations; (2) model development; and (3) automation platform. A brief description of each category is provided in this section.

\section{Data Sources and Recommendations}

Data collected from plant systems contain meta-data related to plant processes, maintenance logs, operator logs, and other relevant items. Typical plant process data relevant to the CWS include gross power, river inlet and outlet temperatures, and motor related information such as on-off duration, motor current, and temperature measurements at the motor stator and bearings. Data collected for the circulating water pump itself include vibration, discharge header pressure, and ambient air temperature. Additional data sets from relevant items such as condenser, river inlet, river outlet, and 
operator actions in the field are extracted and added to the data lake in real-time. A data completeness evaluation will be performed, and recommendations will be provided to the partner utility. Some examples of recommendations include changing the frequency of data collection, installing additional sensors, or replacing/re-calibrating existing sensors.

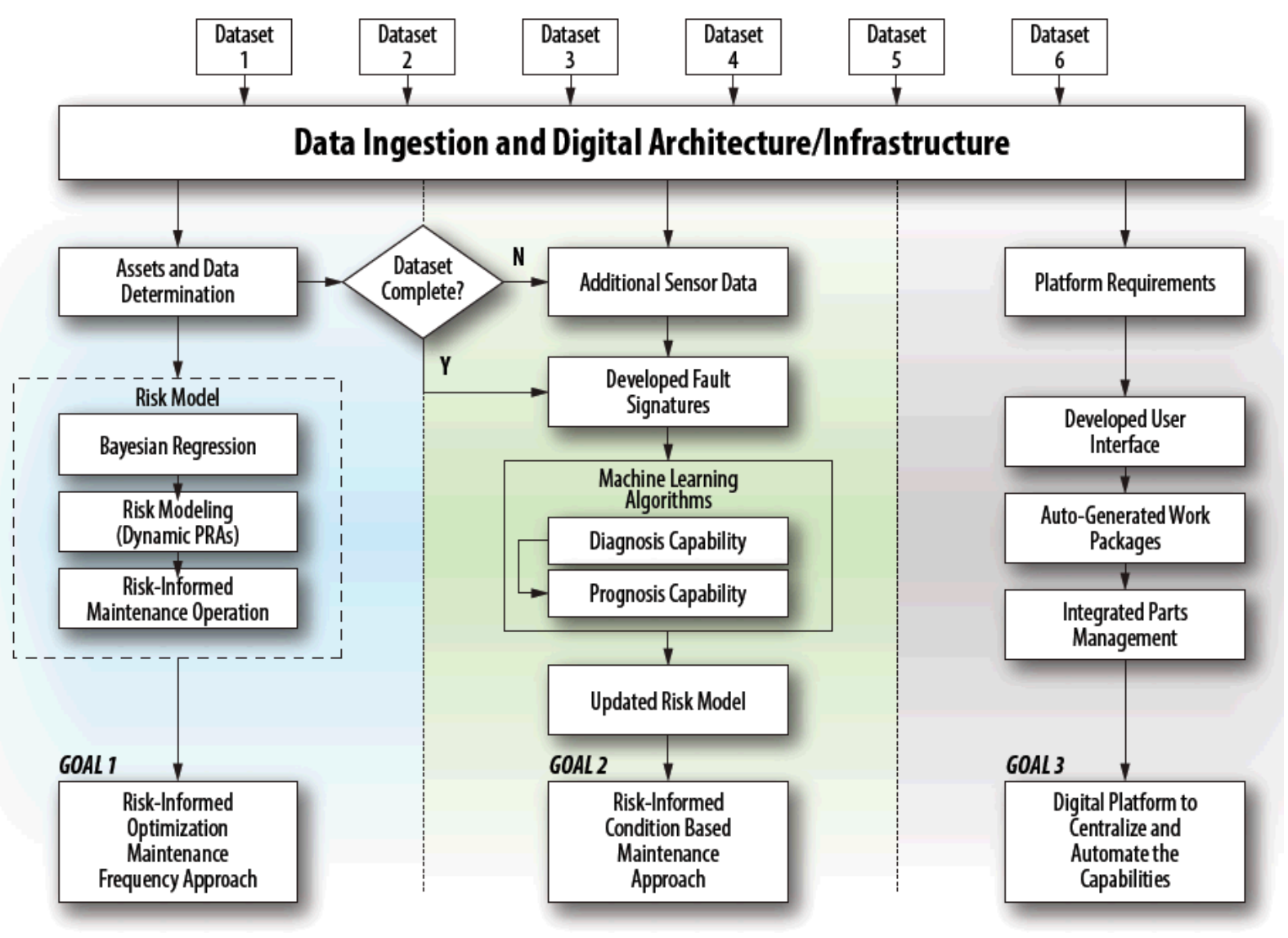

Figure 1. Research scope of integrated risk-informed condition-based predictive maintenance and automated platform.

\section{Model Development}

The research and development activities associated with model development will be performed for risk, diagnostic, and prognostic models, requiring identification of potential failure modes for the target plant system. Risk models calculate component and system risk, utilizing plant-specific historical data obtained in the data sources and recommendation activities. Component failure rates and probability of failure are not fixed in time, but rather evolve, providing a more realistic picture of component and plant risk. For the selected system, Bayesian statistical models are employed to estimate failure rates $(\lambda)$ and probability of failure $(p)$ for components based on their past performance, failures, repair and maintenance, etc. Bayesian statistical models start with known, fixed values of $\lambda$ and $p$ for the components and utilize available data to update $\lambda$ and $p$ based on the most recent information. The models for $\lambda$ and $p$ will be integrated with existing plant-maintained probabilistic risk assessment (PRA) models for the specific system and components, creating dynamic PRA models.

Diagnostic and prognostic models will be developed in a parallel activity, using machine learning (ML) methodologies. ML techniques, including both linear and nonlinear techniques, have shown the ability to correlate the functional relationship present in heterogeneous data and diagnose an evolving fault at an early stage, providing the opportunity to take action and prevent failure. A fault is a particular mode of degradation that can be detected by analyzing plant information. Upon diagnosis 
of a fault, ML techniques will be further utilized to predict progression of the fault under different operating conditions and estimate the remaining useful life of the system or component. The predicted fault growth path and remaining useful life estimation, along with operating condition information, will be utilized to update $\lambda$ and $p$ values, which in turn will update the risk model to make a current risk prediction. The risk prediction is mapped to low, medium, and high risk categories to allow system engineers to make risk-informed decisions on asset maintenance activities, enabling both optimization of maintenance activities and auto generation of work orders.

\section{Development of Automated Platform and Validation}

This part of the research is focused on developing a platform to enable seamless integration of information from risk, diagnostic, and prognostic models with other plant information. Visualization schemes and an effective user interface will be developed that support automatic generation of the necessary electronic work orders by taking into consideration different plant resources such as field worker qualifications, optimal work scheduling, and other aspects. Each stage of the project will be validated via demonstration/testing at the plant as it is achieved. This exercise will enable: (1) evaluation of the effectiveness of the techniques and tools; (2) collection of feedback from plant engineers and maintenance staff to enhance the techniques and tools; and (3) introduction of advanced technologies to provide confidence as PM is gradually phased out.

\section{SUMMARY AND PATH FORWARD}

The project outlines a comprehensive approach for successful research, development, and demonstration of an integrated risk-informed condition-based maintenance capability. Online monitoring and data analytic techniques with advanced risk assessment methodologies are used to schedule maintenance activities without impacting plant operation. The technology-centric approach is designed to reduce preventive maintenance frequency, drive down costs, enhance safety and reliability, and improve the economics of operation in NPPs. This will ensure the nuclear industry as a whole will remain economically competitive and viable in the energy market.

As part of ongoing research, the team is analyzing plant process data, maintenance logs, operator logs, and other relevant data sources to perform a data completeness evaluation for the CWS. The team is also working with a sensor vendor to install wireless vibration sensors on identified locations of the circulating water motors to enable online monitoring capability. 